\title{
dspace.vutbr.cz
}

\section{Analysis and Verification of Identical-Order Mixed-Matrix Fractional-Order Capacitor Networks}

\author{
KARTCI, A.; AGAMBAYEV, A.; HERENCSÁR, N.; SALAMA, K. N.
}

Proceedings of the 2018 14th Conference on PhD Research in Microelectronics and Electronics (PRIME) pp. 277-280

elSBN: 978-1-5386-5387-6

DOI: http://dx.doi.org/10.1109/PRIME.2018.8430316

Accepted manuscript

(C2018 IEEE. Personal use of this material is permitted. Permission from IEEE must be obtained for all other uses, in any current or future media, including reprinting/republishing this material for advertising or promotional purposes, creating new collective works, for resale or redistribution to servers or lists, or reuse of any copyrighted component of this work in other works. KARTCI, A.; AGAMBAYEV, A.; HERENCSÁR, N.; SALAMA, K. N., "Analysis and Verification of Identical-Order Mixed-Matrix Fractional-Order Capacitor Networks", Proceedings of the 2018 14th Conference on PhD Research in Microelectronics and Electronics (PRIME), pp. 277-280, 2018. DOI: 10.1109/PRIME.2018.8430316. Final version is available at https://ieeexplore.ieee.org/document/8430316/ 


\title{
Analysis and Verification of Identical-Order Mixed- Matrix Fractional-Order Capacitor Networks
}

\author{
Aslihan Kartci ${ }^{1}$, Agamyrat Agambayev ${ }^{2}$, Norbert Herencsar ${ }^{1}$, Khaled N. Salama ${ }^{2}$ \\ ${ }^{1}$ Faculty of Electrical Engineering and Communication, Brno University of Technology, Brno, Czech Republic \\ ${ }^{2}$ King Abdullah University of Science and Technology, Thuwal, Saudi Arabia \\ kartci@feec.vutbr.cz, agamyrat.agambayev@kaust.edu.sa, herencsn@feec.vutbr.cz, khaled.salama@kaust.edu.sa
}

\begin{abstract}
In the open literature while capacitors are introduced with $\mathbf{- 9 0}$ degrees phase angle, here we described our fabricated polymer composite, mixed matrix, as a fractionalorder capacitor (FoC). The effect on phase and pseudocapacitance using a detailed numerical and experimental study of circuit network connections of three identical-order FoCs is shown. The used devices have excellent feature such as constant phase angle in the frequency range $200 \mathrm{KHz}-20 \mathrm{MHz}$.
\end{abstract}

Keywords-constant phase element, fractional-order capacitor, FoC, fractional-order circuit, identical-order connection, stability measurement

\section{INTRODUCTION}

The lossy nature of the dielectric material in capacitors and their electrical conductivity does not allow us to treat them as ideal capacitors since their impedance show a complex frequency-dependent behavior. Due to this fact a fractionalorder capacitor (FoC), also called as constant phase element, possess both a real and imaginary impedance part $Z(s)=1 / \omega^{\alpha} C_{\alpha}\left[\cos \left(\frac{\alpha \pi}{2}\right)+j \sin \left(\frac{\alpha \pi}{2}\right)\right]$ while its phase is frequency independent that differs from the series connected resistor and capacitor. However, an ideal capacitor has only an imaginary part [1], as demonstrated in Fig. 1. This is particularly important, if the proposed application requires a configuration using capacitors, where errors accumulate the metrics of the individual components. Thus, applications of FoCs are geared towards engineering in many application areas. Particularly, the research was focused on improvement of peaking in passband of filters [2], increase of oscillation frequency [3], electronic tunability of resonators [4], [5], modelling fractional-order impendance of neural systems [6] or employing a real effective capacitances by using a model consisting a FoC in energy storage devices [7]. Recently, a ferroelectric polymer [8] and carbon nanotube-polymer composite [9] based FOCs were introduced. Their easy integration on printed circuit board and constant phase angle are two main advantages of these fabricated FoCs.

The circuit network connections of FoCs and the order of each element have a crucial role in calculating the equivelant impedence in supercapacitor bank design [7]. Therefore, this paper aims to study the equivalent magnitude, phase, and pseudo-capacitance

The research described in this paper was financed by the National Sustainability Program under grant LO1401 and by the Czech Science Foundation under grant no. 16-06175S. For the research, an infrastructure of the SIX Center was used.

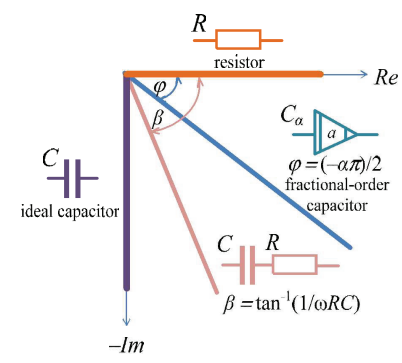

Fig. 1. Nyquist diagram of fractional-order capacitor.

of three identical-order series- and parallel-connected fabricated polymer composite FoCs in the fractional domain. The phase is found to be constant with \pm 4 degrees phase angle deviation in the measured frequency range of interest $200 \mathrm{KHz}-20 \mathrm{MHz}$. Moreover, similarly to given single FoCs, the pseudo-capacitance in the parallel connection was higher than those of series-connected FoCs, which agrees well with the related integer-order theory.

\section{FABRICATED FOCS}

The behavior of three samples of fabricated ferroelectric polymer (TP, P) and carbon nanotube-polymer (CP) based FoCs of different orders (respectively $\alpha=\{0.69,0.92,0.62$ ) was confirmed using an Impedance Analyzer. The magnitude, phase, and pseudo-capacitance at center frequency $f_{\mathrm{c}}=2 \mathrm{MHz}$ of all the devices are $\{2.24,6.43,1.51\} \mathrm{k} \Omega,\{-61.86,-82.59$, $-55.45\}$ degrees, and $\{5.89 \mathrm{n}, 47.52 \mathrm{p}, 28.1 \mathrm{n}\} \mathrm{Farad} \cdot \mathrm{sec}^{\alpha-1}$, respectively. An illustration of fabricated PCB compatible fabricated FoC and its cross- sectional SEM image are shown in Fig. 2. Each device contains nine FoCs with identical order and pseudo-capacitance.

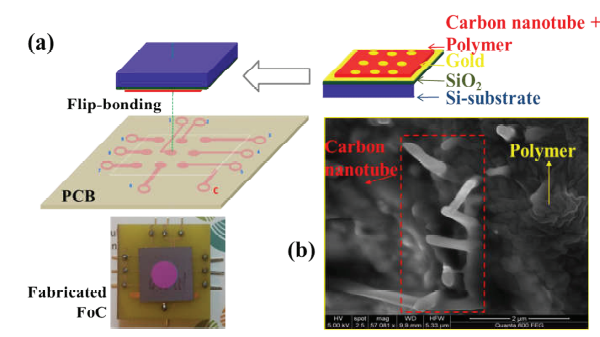

Fig. 2. (a) PCB compatible FoC from carbon nanotube-polymer, (b) its crosssectional SEM image. 
TABLE I.

Impedance, MAgnitude And Phase Descriptions Of ThreE SERIES-CONNECTEd FoCs.

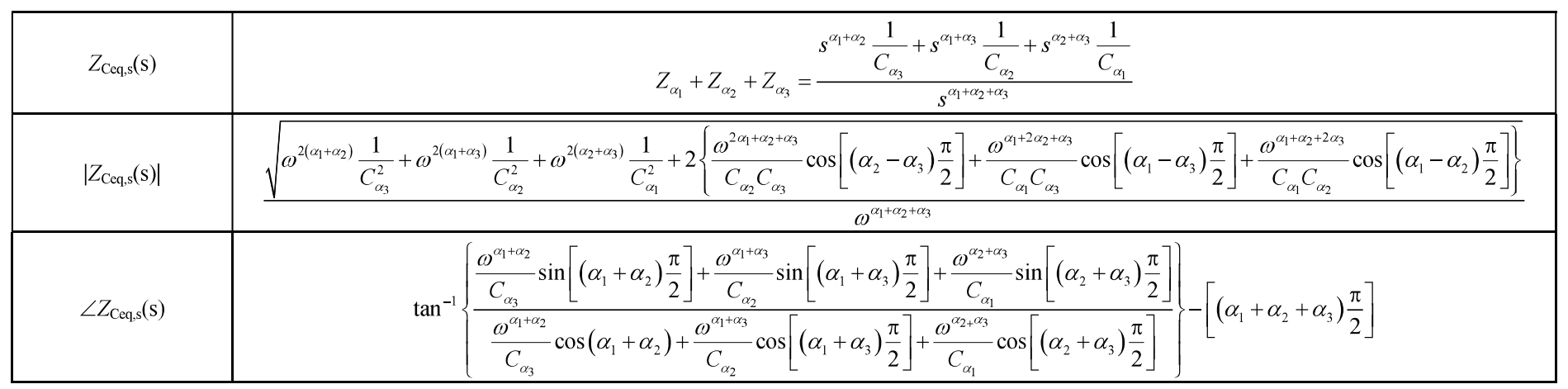

\section{CASE STUdies AND THEIR EXPERIMENTAL VERIFICATION}

This section introduces to the literature specific case studies based on general approach previously presented by the authors [10]. In this paper for the first time three identical-order solidstate passive FoCs are connected in series, parallel, and their special inter-connection. During experiments via impedance analyzer, the devices introduced in Section II were used with applied $500 \mathrm{mV}$ AC voltage in the frequency range $200 \mathrm{KHz}-20 \mathrm{MHz}$. A sinsoidal input of frequency of $1 \mathrm{MHz}$ was applied, while the common node of FoCs was grounded $\left(V_{\mathrm{g}}=0 \mathrm{~V}\right)$.

\section{A. Connection of FoCs in Series}

Considering three series-connected FoCs, as shown in Figs. 3, and assuming each with orders $\alpha_{1}, \alpha_{2}, \alpha_{3}$, and impedances $Z_{\alpha_{1}}(s)=1 / s^{\alpha_{1}} C_{\alpha_{1}}, \quad Z_{\alpha_{2}}(s)=1 / s^{\alpha_{2}} C_{\alpha_{2}}, \quad$ and $Z_{\alpha_{3}}(s)=1 / s^{\alpha_{3}} C_{\alpha_{3}}$, the equivalent impedance, magnitude, and phase responses can be calculated from the equations in Table I. It is clear that the order of each element has a significant effect on the impedance of each $\mathrm{FoC}$ and their equivalent capacitance cannot be considered. Therefore, to verify the theoretical analysis, the behavior of the three identical FoCs has been verified experimentally. The phase, magnitude, and pseudo-capacitance responses of the equivalent impedances $Z_{\text {Ceq,s }}(s)$ are shown in Fig. 4 . For reference, both pseudo-capacitance and phase for an individual FoC have been plotted inside same figures. Compared to a single device, we note that the phase response of three identical order FoCs connected in series remains same. However, its magnitude of the equivalent impedance is tripled while pseudo-capacitance is one-third as shown in the inset of Fig. 4(a), and Fig. 4(b).

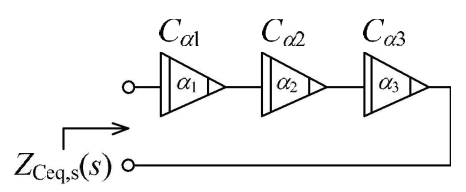

Fig. 3. Series-conneted three FoCs.
TABLE II. COMPARISON OF IDENTICAL-ORDER SERIES-CONNECTED FoCs: MEASURED AND CALCULATED RESUlTS.

\begin{tabular}{|c|c|c|}
\hline No. & $\# \mathbf{1}$ & $\boldsymbol{\# 2}$ \\
\hline Connection of Orders & $0.69+0.69+0.69$ & $0.92+0.92+0.92$ \\
\hline Equivalent Impedance $@ f_{\mathrm{c}}[\mathrm{k} \Omega]$ & $6.58(6.65)$ & $10.54(22.09)$ \\
\hline Phase $\left[{ }^{\circ}\right]$ & $-60.26(-62.13)$ & $-83.99(-81.64)$ \\
\hline Relative Phase Error [\%] & -3.00 & 2.88 \\
\hline Equivalent Order $\alpha[-]$ & $0.67(0.69)$ & $0.93(0.91)$ \\
\hline Pseudo-Capacitance $\left[\right.$ Farad $\left.\cdot \mathrm{sec}^{\alpha-1}\right]$ & $2.68(1.89) \mathrm{n}$ & $22.48(16.46) \mathrm{p}$ \\
\hline
\end{tabular}
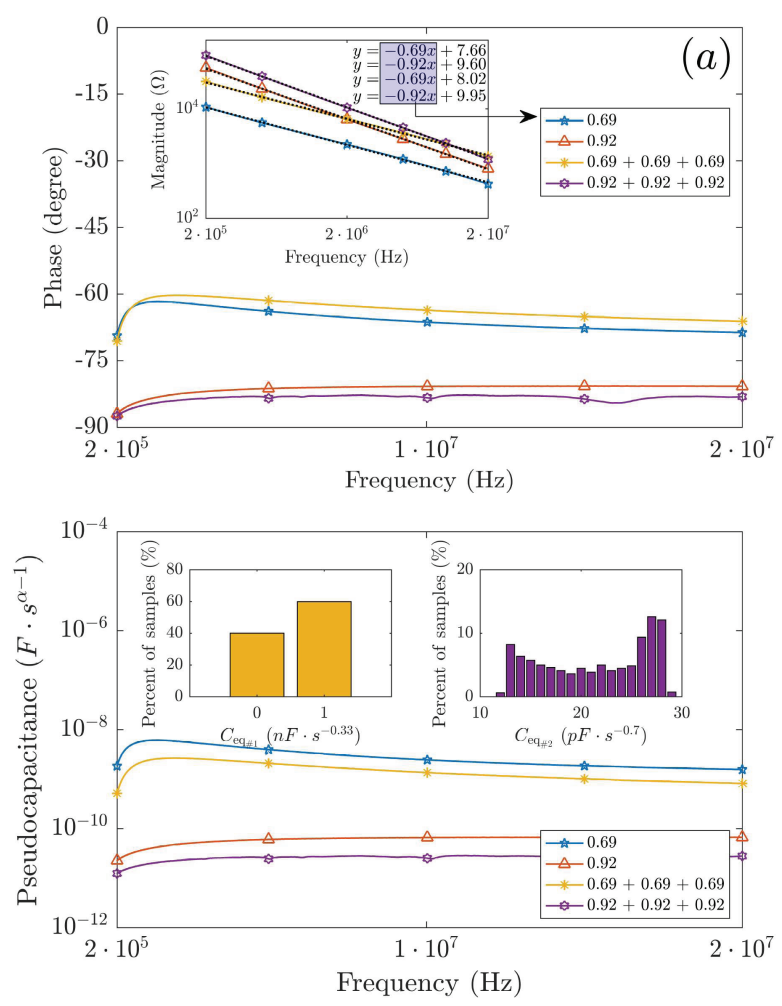

Fig. 4. Experimental verification of three series-connected FoCs: (a) phase, magnitude, (b) pseudo-capacitance responses. 
TABLE III. IMPEDANCE, MAGNITUde AND PhASE DESCRIPTIONS OF THREE PARALlEL-CONNECTED FoCs.

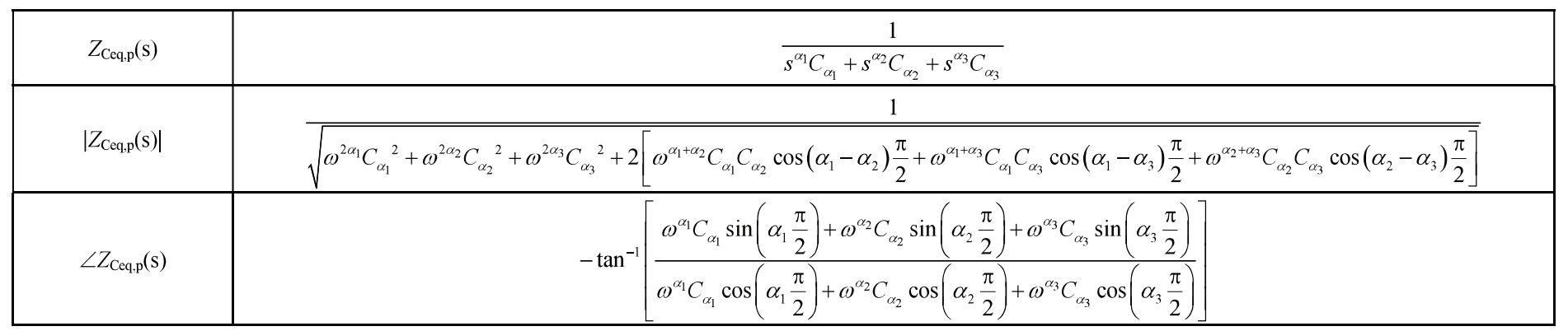

The comparison of measured values @ $f_{\mathrm{c}}=2 \mathrm{MHz}$ and expected results, i.e, calculated via MATLAB are evaluated in Table II. For cases \#1 $\rightarrow \# 2$ given in Table II, equivalent magnitudes vary in ranges $(31.79 \rightarrow 1.36) \mathrm{k} \Omega$ and $(95.20 \rightarrow 1.19) \mathrm{k} \Omega$, respectively. The table also includes calculated relative phase error and corresponding pseudocapacitance of each connection. The magnitude and pseudocapacitance responses are plotted in the logarithmic scale meanwhile the phase is in linear scale. Moreover, to estimate the equivalent order $\alpha$ (or phase), the measured magnitude data are fitted to the function $\log |Z|=\alpha \log f+\log (2 \pi)^{\alpha} C_{\alpha}$ using the linear least squares (LLS) method. The obtained equivalent equations from fitting the magnitude is equal to measurement samples that are provided inside Fig. 4(a). As a result, the orders of single devices TP, P2, i.e. $0.69,0.92$ with corresponding phases $-61.86,-82.59$, [degrees] are evidently respond to their equivalent orders from series connections that are found to be $0.67,0.93$ (corresponding to Table II cases $\# 1 \rightarrow \# 2$ with phases $-60.26,-83.99$ ).

\section{B. Connection of FoCs in Parallel}

In case of parallel connection of three identical-order FoCs as in Fig. 5, the equivalent impedance, magnitude and phase responses can be expressed as in Table III. In order to demonstrate the behavior of an equivalent FoC with impedance $Z_{\text {Ceq,p, }}$, the phase, magnitude and pseud-capacitance responses was experimentally verified. The obtained measurement results are shown in Fig. 6 while the comparison of measured values @ $f_{\mathrm{c}}=2 \mathrm{MHz}$ and calculated results via MATLAB are listed in Tables IV, respectively. Inspecting the obtained results, it is evident that the phase remains identical to initial single FoCs phase and the only change is in the magnitude response, which reflects the pseudo-capacitance $C_{\alpha}$. Obviously, the magnitude is the one-third of individual FoC. The equivalent orders, which are obtained using the LLS fitting and given in Fig. 6(a) as an inset, are found to be 0.69 and 0.92 as equal to related $\mathrm{FoC}$ order. The calculated relative phase errors for these cases are $-1.63 \%$ and $-0.08 \%$. It is worth to note that the accuracy of above theoretical analyzes are verified and showed a flexibility and degree of freedom to work with any order of FoCs with a random connection.
TABLE IV. COMPARISON OF IDENTICAL ORDER PARALlEL CONNECTED FOCS: MEASUREMENT AND CALCULATED RESULTS.

\begin{tabular}{|c|c|c|}
\hline No. & $\# \mathbf{3}$ & $\boldsymbol{\# 4}$ \\
\hline Connection of Orders & $0.69\|0.69\| 0.69$ & $0.92\|0.92\| 0.92$ \\
\hline Equivalent Impedance @ $f_{\mathrm{c}}[\mathrm{k} \Omega]$ & $0.700(0.737)$ & $2.30(2.34)$ \\
\hline Phase $\left[{ }^{\circ}\right]$ & $-61.12(-62.13)$ & $-82.14(-82.20)$ \\
\hline Relative Phase Error [\%] & -1.63 & -0.08 \\
\hline Equivalent Order $\alpha[-]$ & $0.68(0.69)$ & $0.91(0.91)$ \\
\hline Pseudo-Capacitance $\left[\right.$ Farad $\left.\cdot \mathrm{sec}^{\alpha-1}\right]$ & $21.55(17.04) \mathrm{n}$ & $144.45(140.23) \mathrm{p}$ \\
\hline
\end{tabular}

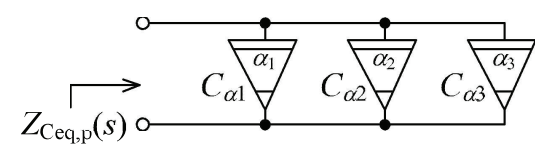

Fig. 5. Parallel-conneted three FoCs.
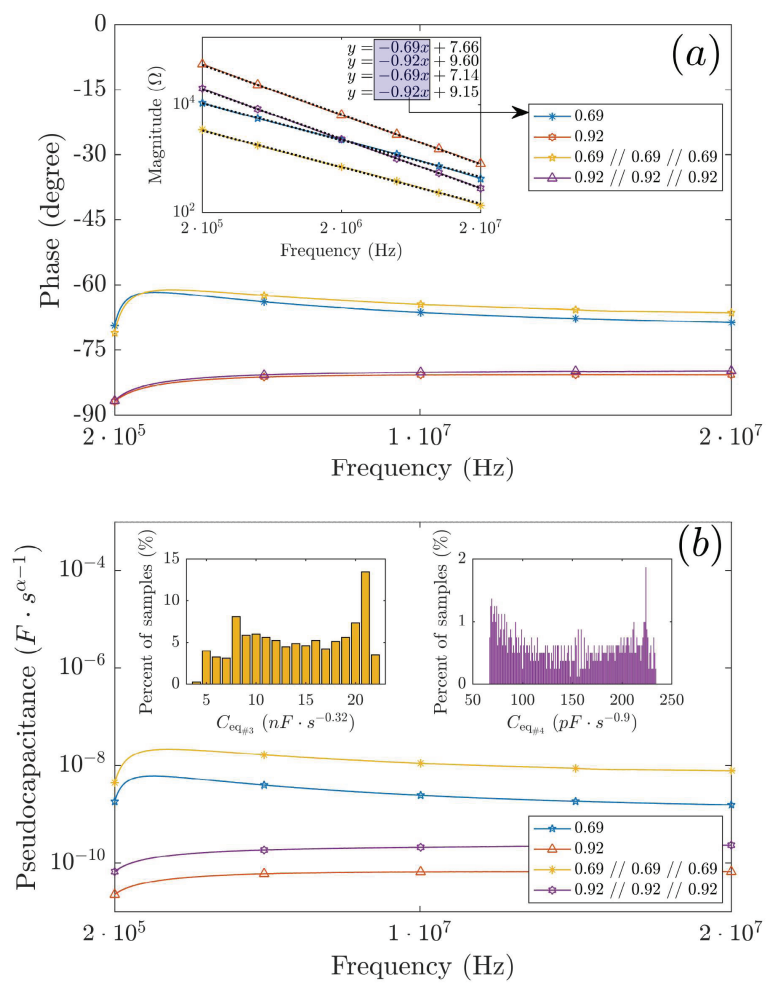

Fig. 6. Experimental verification of three parallel-connected FoCs: (a) phase, magnitude, (b) pseudo-capacitance responses. 
I ABLE V. COMPARISON OF INTER-CONNECTED FOLS: MEASURED AND CALCUlATED RESUlts.

\begin{tabular}{|c|c|}
\hline No. & \#5 \\
\hline Connection of Orders & $\begin{array}{c}(0.69 \| 0.69)+(0.92 \| 0.92)+ \\
(0.62 \| 0.62)\end{array}$ \\
\hline Equivalent Impedance $@ f_{\mathrm{c}}[\mathrm{k} \Omega]$ & $7.23(4.96)$ \\
\hline Phase $\left[{ }^{\circ}\right]$ & $-76.09(-73.94)$ \\
\hline Relative Phase Error $[\%]$ & 2.90 \\
\hline Equivalent Order $\alpha[-]$ & $0.85(0.82)$ \\
\hline Pseudo-Capacitance $\left[\right.$ Farad $\left.\cdot \sec ^{\alpha-1}\right]$ & $137.72(196.33) \mathrm{p}$ \\
\hline
\end{tabular}

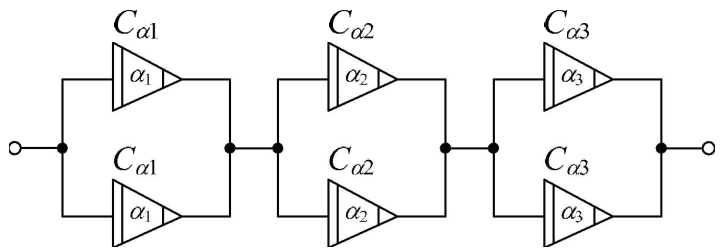

Fig. 7. Inter-connection (series-parallel) of FoCs.
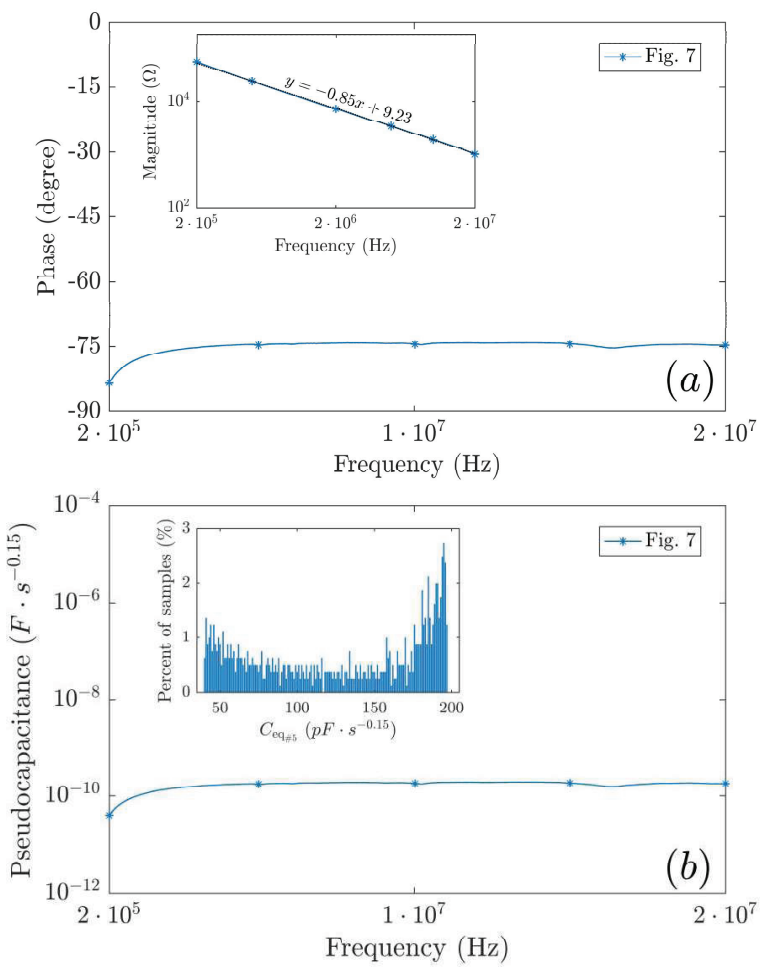

Fig. 8. Experimental verification of inter-connected FoCs: (a) phase, magnitude, (b) pseudo-capacitance responses.

\section{Inter-connection of FoCs}

Considering the network shown in Fig. 7, its equivalent impedance is given as:

$Z_{\text {eq\#5 }}(s)=\frac{2 s^{\alpha_{1}+\alpha_{2}} C_{\alpha_{1}} C_{\alpha_{2}}+2 s^{\alpha_{1}+\alpha_{3}} C_{\alpha_{1}} C_{\alpha_{3}}+2 s^{\alpha_{2}+\alpha_{3}} C_{\alpha_{2}} C_{\alpha_{3}}}{s^{\alpha_{1}+\alpha_{2}+\alpha_{3}} C_{\alpha_{1}} C_{\alpha_{2}} C_{\alpha_{3}}}$.

The measured phase, magnitude, and pseudo-capacitance responses of the inter-connected FoCs are shown in Fig. 8 and the detailed comparison of results@2 MHz are given in Table V. The calculated relative phase error and equivalent order of interconnection are $+2.90 \%$ and 0.85 , respectively. It is clear that small deviation in phase effects on pseudocapacitance lot due to the power of order. By simplifying Eq. (1) with assuming $\alpha_{1}=\alpha_{2}=\alpha_{3}$, then the equivalent impedance would be $2 C_{\alpha} / 3$.

\section{CONCLUSION}

The stability measurement of mixed-matrix identical-order FoC connections and the effect of frequency on the capacitive performance of the devices were studied. This is due to the change of the order (dispersion parameter) $\alpha$ from the high frequency (more resistive) to a low-frequency region (more capacitive)which results in the proper use of FoCs, either individually or more importantly in FoCs connections. It was also shown that $C_{\alpha}$ match well with classical circuit theory formulae (equivalent capacitance of series- or parallelconnected FoCs, identical order phase, magnitude, and pseudo-capacitance) expected by application designers. As future work, the experimental results of series- and parallelconnected arbitrary- and identical-order FoCs will be correlated with dielectric properties in different microstructures. Therefore, the FoC characteristics with connection of different dielectric materials will be the key point. Moreover, these fabricated FoCs will be modelled with different structures and optimized using different algorithms as the next step. This study is the very first step to achieve understanding of natural behavior of FoCs and move forward to computational part.

\section{REFERENCES}

[1] S. Westerlund and L. Ekstam, "Capacitor theory," IEEE Transactions on Dielectrics and Electrical Insulator, vol. 1, pp. 826-839, 1994.

[2] A. Adhikary, S. Sen, and K. Biswas, "Practical realization of tunable fractional order parallel resonator and fractional order filters," IEEE Trans. on Circuits and Systems I; vol. 63, pp. 1142-1151, 2016.

[3] A. Kartci, N. Herencsar, J. Koton, L. Brancik, K. Vrba, G. Tsirimokou, C. Psychalinos, "Fractional-Order Oscillator Design Using Unity-Gain Voltage Buffers and OTAs," In Proc. 60th IEEE Int. Midwest Symp. Circuits Syst. (MWSCAS), Boston, MA, USA, pp. 555-558, 2017.

[4] G. Tsirimokou, C. Psychalinos, A. S. Elwakil, and K. N. Salama, "Electronically tunable fully integrated fractional-order resonator," IEEE Trans. on Circuits and Systems II, vol. 65, pp.166-170, 2018.

[5] L. Kadlčík and P. Horský, "A CMOS Follower-Type Voltage Regulator With a Distributed-Element Fractional-Order Control," IEEE Trans. on Circuits and Systems I, in print, pp. 1-11, 2018.

[6] C. M. Ionescu, "Emerging tools in engineering: fractional order ladder impedance models for respiratory and neural systems," IEEE Journal on Emerging and Selected Topics in Circuits and Systems,vol. 3, pp. 42531,2013 .

[7] A. Allagui, A. S. Elwakil, B. J. Maundy, and T. J. Freeborn, "Spectral capacitance of series and parallel combinations of supercapacitors," ChemElectroChem, vol. 3, no. 9, pp. 1429-1436, 2016.

[8] A. Agambavev, S. Patole, M. Farhat, A. Elwakil, H. Bagci, and K. N. Salama, "Ferroelectric fractional-order capacitors," ChemElectroChem, vol. 4, pp. 2807-2813, 2017

[9] A. Agambayev, K. H. Rajab, A. H. Hassan, M. Farhat, H. Bagci, and K. N. Salama, "Towards fractional-order capacitors with broad tunable constant phase angles: Multi-walled carbon nanotube-polymer composite as a case study," J. of Physics D: App. Physics, vol. 51, pp. 1-6, 2018.

[10] A. Kartci, A. Agambayev, N. Herencsar, and K.N. Salama, "Series-, parallel-, and inter-connection of solid-state arbitrary fractional-order capacitors: theoretical study and experimental verification," IEEE Access, vol. 6, pp. 10933-10943, 2018. 